\title{
MicroRNA-196a2 single nucleotide polymorphism rs I I6I49/3 in Egyptian patients with chronic lymphocytic leukemia
}

\begin{abstract}
Background: MicroRNAs are recently identified class of small single stranded RNA molecules of 21-23 nucleotides that regulate gene expression at the posttranscriptional level. Chronic lymphocytic leukemia (CLL) is characterized by a typical defect in apoptosis resulting in accumulation of mature resting B cells within the peripheral blood, bone marrow and lymphoid organs. One of the relevant microRNAs to CLL is microRNA-196a2, as its potential targets of action include genes involved in apoptosis regulation. The single nucleotide polymorphism (SNP) in microRNA-196a2 rs $11614913 \mathrm{C} / \mathrm{T}$ has been implicated as possible biomarker associated with multiple kinds of cancers. While multiple recent studies showed that genetic polymorphisms play an influential role in CLL susceptibility, the role of microRNA-196a2 SNP rs11614913in CLL remains elusive.
\end{abstract}

Patients and methods: The present study was conducted on 40 newly diagnosed Egyptian CLL patients and 40 control subjects. Genotyping of microRNA-196a2 SNP rs 11614913 was performed by real-time PCR.

Results: The CC genotype was found in 55\% of CLL patients versus $12.5 \%$ of the controls. On the other handthe TT and TC genotypes were significantly predominant in the control group compared to CLL patients with frequencies of $25 \%$ and $62.5 \%$ versus $10 \%$ and $35 \%$ respectively $(\mathrm{p}<0.001)$. The $\mathrm{C}$ allele carriers were found to have 3.38 fold increased risk for development of CLL $(\mathrm{OR}=3.38, \mathrm{CI}=1.75-6.56)$ while the $\mathrm{T}$ allele seems to be protective against $\mathrm{CLL}(\mathrm{OR}=0.29, \mathrm{CI}=0.15-0.57)$.

Conclusion: We could conclude that the CC genotype and the $\mathrm{C}$ allele of miR-1962a SNP rs3217927are implicated in the risk of CLL development in Egyptian patients while the $\mathrm{T}$ allele has a protective effect. However, additional well-designed large studies are required for the validation of these associations

Keywords: MicroRNA-196a2, SNP, rs11614913, CLL
Volume 7 Issue I - 2019

\author{
Akram Deghady,' Reham Abo Elwafa,' Manal \\ AE Lsorady, ${ }^{2}$ Darine Elbana \\ 'Clinical Pathology Department,Alexandria University, Egypt \\ ${ }^{2}$ Hematology Medicine Department, Alexandria University, Egypt
}

Correspondence: Akram Deghady, Clinical Pathology Department, Faculty of Medicine, Alexandria University, Alexandria, Egypt, Email akram 196I@hotmail.com

Received: February 20, 2019 | Published: February 28, 2019

\section{Introduction}

MicroRNAs (miRNAs) are small, single-stranded, 19-21 nucleotide long non-protein-coding RNA molecules. They have the capacity for simultaneous regulation of tens to hundreds of genes through direct targeting of 3' untranslated regions (3'UTR) resulting in either degradation of mRNA or inhibition of translation. MiRNAs are important to several fundamental biological processes including proliferation, apoptosis, development, and cellular differentiation. ${ }^{1}$

The dysregulation of miRNAs expression in several malignancies as well as their frequent location in fragile chromosomal regions support their relevance to the development and pathogenesis of malignancies. They have the capacity to function as either oncogenes or tumor suppressors. These small RNAs could represent novel cancer biomarkers.

Single nucleotide polymorphisms (SNPs) are the most common type of genetic variations associated with population diversity, disease susceptibility and individual response to treatment. ${ }^{3}$ MiRNA expression levels can be altered by SNPs located in miRNA-related regions. These SNPs modify miRNA production as well as the target binding affinity and specificity and subsequently miRNA function. Therefore, SNPs of miRNAs have begun to draw attention, due to their critical regulatory role in cancer progression. ${ }^{4}$ In addition, several studies have described polymorphisms in miRNAs associated with the susceptibility to different malignancies as esophageal, breast, lung and gastric cancers. ${ }^{3}$

MicroRNA-196a (miR-196a) gene is located at a region between HOXC10 and HOXC9 on chromosome 12 (12q13.13). The miR$196 \mathrm{a} 2$ SNP rs11614913 lies in the mature sequence of miR-196a2 and negatively impacts endogenous processing of miRNA precursor to its mature form. Several studies investigated this genetic variant as possible biomarker associated with multiple cancers. ${ }^{5}$

Up-regulation of miR-196a has been documented in various types of human cancer and leukemia. To date several reports have described altered expression of miR-196a2 in CLL, suggesting its implication in the pathogenesis of the disease. ${ }^{6}$ Indeed, variations in miRNA transcripts have been seen to be common in CLL, suggesting that CLL predisposition involves genetic alterations in miRNAs. ${ }^{7}$ However, despite accumulating evidence that inherited genetic variation in miRNA genes can contribute to the predisposition and prognosis for cancer, their role in CLL has not been extensively studied. In this context, the aim of the present work was to investigate the miR-196a2 SNP rs11614913 contribution to the risk of CLL in Egyptian patients.

\section{Patients and methods}

The current study was conducted on 80 subjects; 40 patients of both sexes newly diagnosed with CLL admitted to Alexandria Main 
University Hospital and a control group of 40 healthy subjects with matched age and sex. The clinico-pathological data of the study subjects are summarized in Table 1. Diagnosis of CLL was established based on the current World Health Organization (WHO) classification guidelines.CLL patients with a second malignancy, received prior therapy or with relapsed CLL were all excluded from the study. All study participants had given an informed consent after explaining the nature, steps, and aim of the study. The study was conducted with the approval of the Medical ethics committee of Alexandria Faculty of Medicine. Peripheral blood samples were obtained from both CLL patients and controls on potassium ethylenediamine tetra acetic acid (K3 EDTA).

Table I Characteristics of the study participants

\begin{tabular}{|c|c|c|c|}
\hline & $\begin{array}{l}\text { CLL patients } \\
(n=40)\end{array}$ & $\begin{array}{l}\text { Controls } \\
(n=40)\end{array}$ & $\mathbf{p}$ \\
\hline \multicolumn{4}{|l|}{ Sex } \\
\hline Male & $22(55 \%)$ & $24(60 \%)$ & \multirow{2}{*}{0.651} \\
\hline Female & $18(45 \%)$ & $16(40 \%)$ & \\
\hline Age (years) & $59.05 \pm 7.90$ & $57.75 \pm 6.59$ & 0.427 \\
\hline WBCs $\left(\times 10^{9} / \mathrm{L}\right)$ & $27.95(9.80-82.0)$ & $8.10(4.40-9.70)$ & $<0.001^{*}$ \\
\hline $\mathrm{Hb}(\mathrm{g} / \mathrm{dL})$ & $11.54 \pm 2.28$ & $13.63 \pm 1.49$ & $<0.001^{*}$ \\
\hline Platelets $\left(\times 10^{9} / \mathrm{L}\right)$ & $189.0(30.0-410.0)$ & $\begin{array}{l}295.50(211.0- \\
473.0)\end{array}$ & $<0.001^{*}$ \\
\hline Lymphocytes (\%) & $66.30 \pm 10.17$ & $30.45 \pm 8.64$ & $<0.001^{*}$ \\
\hline $\operatorname{ALC}\left(\times 10^{9} / \mathrm{L}\right)$ & $16.30(6.0-61.70)$ & $2.30(1.20-2.90)$ & \\
\hline \multicolumn{4}{|l|}{ Rai staging system } \\
\hline 0 & $1(2.5 \%)$ & - & \\
\hline I & $9(22.5 \%)$ & - & \\
\hline II & $16(40.0 \%)$ & & \\
\hline III & $9(22.5 \%)$ & - & \\
\hline IV & $5(12.5)$ & - & \\
\hline \multicolumn{4}{|l|}{ Splenomegally } \\
\hline No & $16(40 \%)$ & - & \\
\hline Yes & $24(60 \%)$ & - & \\
\hline \multicolumn{4}{|l|}{ hepatomegaly } \\
\hline No & $34(85 \%)$ & - & \\
\hline Yes & $6(15 \%)$ & - & \\
\hline \multicolumn{4}{|l|}{ lymphadenopathy } \\
\hline No & $12(30 \%)$ & - & \\
\hline Yes & $28(70 \%)$ & - & \\
\hline \multicolumn{4}{|l|}{$\begin{array}{l}\text { rs11614913 } \\
\text { genotypes }\end{array}$} \\
\hline TT & $4(10 \%)$ & $10(25 \%)$ & \\
\hline $\mathrm{TC}$ & $14(35 \%)$ & $25(62.5 \%)$ & \\
\hline $\mathrm{CC}$ & $22(55 \%)$ & $5(12.5 \%)$ & $<0.001^{*}$ \\
\hline \multicolumn{4}{|l|}{ rs11614913Alleles } \\
\hline $\mathrm{T}$ & $22(27.5 \%)$ & $45(56.3 \%)$ & \\
\hline $\mathrm{C}$ & $58(72.5 \%)$ & $35(43.8 \%)$ & $<0.001^{*}$ \\
\hline
\end{tabular}

\section{MicroRNA- | 96a2 SNP rs I I 6 | 49 | 3genotyping}

Genomic DNA extraction was done by standard protocol using QIAamp DNA Blood Mini Kit (QIAGEN, USA). The quantity (ng/ $\mu \mathrm{l})$ and the purity of the extracted DNA were measured on nanodrop 2000 ((Thermo Scientific, USA). The ratio of absorbance at 260/280 and $260 / 230$ of $1.8 \pm 0.2$ indicated highly pure DNA. The miR-196a2 SNP rs11614913 genotyping was carried out on Rotor-Gene Q Real time PCR system(QIAGEN, Germany) using TaqMan SNP genotyping assay (Applied Biosystems, USA). The 40xSNP Genotyping Assays was diluted to a 20x working stock solution, of which $1 \mathrm{ul}$ was added to $10 \mu 1$ Universal TaqMan Master Mix and $2 \mu 1$ of the extracted genomic DNA in a reaction total volume of $20 \mu 1$. The thermal profile used was $95^{\circ} \mathrm{C}$ for $5 \mathrm{~min}$, followed by 40 cycles of $95^{\circ} \mathrm{C}$ for 15 seconds, and $60^{\circ} \mathrm{C}$ for $1 \mathrm{~min}$

\section{Statistical analysis}

Statistical analysis datawas fed to the computer and analyzed using IBM SPSS software package version 20.0. (Armonk, NY: IBM Corp). Qualitative data was described using number and percent. Quantitative data was described using range (minimum and maximum), mean, standard deviation, and median. The significance of the results obtained was judged at the $5 \%$ level.

\section{Results}

\section{Characteristics of the study subjects}

Subjects' characteristics are summarized in Table 1. There was no significant difference in the distribution of both groups according to age and sex. Comparison of the $\mathrm{CBC}$ parameters revealed significantly lower $\mathrm{Hb}$ level and platelet count in patients with CLL compared to the controls. The WBCs count and the absolute lymphocytic counts were significantly higher in the CLL patients. Most of the studied patients with CLL (75.0\%) were diagnosed incidentally during routine blood examination for other reasons, while the remainder $25 \%$ was diagnosed after having symptoms of infection or anemia. Regarding the Rai staging system, higher percentage for stage II disease (40.0\%) followed by stage I and III ( $22.5 \%$ for each), $12.5 \%$ in stage IV and $2.5 \%$ in stage 0 .

\section{Association between miR-|96a2 SNP rs I |6 |49|3 and CLL risk}

The distribution of miR-196a2 SNP rs11614913 genotype in patients with CLL and the controls was in accordance with the Hardy-Weinberg equilibrium. There was a statistically significant difference in the genotypes distribution of the miR-196a2 SNP rs 11614913 between CLL patients and controls. In the control group the genotypic frequencies were as follows: $10(25 \%)$ were TT genotype, $25(62.5 \%)$ were TC and 5(12.5\%) were CC. In patients with CLL, 4 (10\%) were TT genotype, 14 (35\%) were TC and 22 $(55 \%)$ were CC. With regard to the allelic frequency, the $\mathrm{C}$ allele was significantly higher in patients with CLL compared to the controls with a predominance of $72.5 \%$ and $43.8 \%$ respectively (Table 1 ). The odds ratio was calculated at $95 \%$ Confidence Interval and showed that the $\mathrm{C}$ allele carriers have 3.38 fold increased risk for development of CLL compared to the $\mathrm{T}$ allele carriers $(\mathrm{OR}=3.38, \mathrm{CI}=1.75-6.56)$ while the $\mathrm{T}$ allele seems to be protective against CLL development $(\mathrm{OR}=0.29, \mathrm{CI}=0.15-0.57)$ (Table 2).

*: Statistically significant at $\mathrm{p} \leq 0.05$. ALC, absolute lymphocytes count 
Table 2 Comparison between the two studied groups according to miR-196a2 rs I I6I49I3 genotype and allele frequencies

\begin{tabular}{|c|c|c|c|c|c|c|c|}
\hline & \multicolumn{2}{|c|}{ Cases } & \multicolumn{2}{|c|}{ Control } & \multirow{2}{*}{ OR } & \multirow{2}{*}{$\mathbf{p}$} & \multirow{2}{*}{$\begin{array}{l}\text { 95\% C.I } \\
\text { L.L-U.L }\end{array}$} \\
\hline & No. & $\%$ & No. & $\%$ & & & \\
\hline Genotypes frequency & \multicolumn{2}{|c|}{$(n=40)$} & \multicolumn{2}{|c|}{$(n=40)$} & & & \\
\hline TT & 4 & 10.0 & 10 & 25.0 & 0.33 & 0.087 & $0.09-1.17$ \\
\hline $\mathrm{TC}$ & 14 & 35.0 & 25 & 62.5 & $0.32^{*}$ & $0.015^{*}$ & $0.13-0.80$ \\
\hline $\mathrm{CC}$ & 22 & 55.0 & 5 & 12.5 & $8.56^{*}$ & $<0.001^{*}$ & $2.78-26.36$ \\
\hline Allele frequency & \multicolumn{2}{|c|}{$(n=80)$} & \multicolumn{2}{|c|}{$(n=80)$} & & & \\
\hline $\mathrm{T}$ & 22 & 27.5 & 45 & 56.3 & $0.29^{*}$ & $<0.001^{*}$ & $0.15-0.57$ \\
\hline C & 58 & 72.5 & 35 & 43.8 & $3.38^{*}$ & $<0.001^{*}$ & $1.75-6.56$ \\
\hline
\end{tabular}

$\chi^{2}, \mathrm{p}: \chi^{2}$ and $\mathrm{p}$ values for Chi square test for comparing between the two groups

OR, Odd's ratio; L.L, Lower limit; U.L, Upper limit; C.I, Confidence interval

*: Statistically significant at $\mathrm{p} \leq 0.05$

\section{Discussion}

Chronic lymphocytic leukemia (CLL) has been reported to have the highest genetic predisposition of all hematological malignancies. ${ }^{(8)}$ It is a disease of defective apoptosis resulting in accumulation of mature resting $\mathrm{B}$ cells in the peripheral blood, bone marrow and other lymphoid organs. The miR-1962a SNP rs11614913 is located in the $3 p$ mature miRNA region, so it could affect maturation of $5 p$ and $3 p$ miRNAs, as well as the expression and function of these miRNAs. In the miR-1962a SNP $\mathrm{T}<\mathrm{C}$ rs 11614913, theC allele led to less efficient processing of the miRNA precursor to its mature forms as well as diminished capacity to regulate target genes. ${ }^{9}$ Interestingly, the potential targets of miR196a2 action include genes with relevance in CLL, such as CDKN1B or HMOX1. Therefore, alterations in the sequence of miR196a2 may affect miRNA function and could contribute to CLL development and pathogenesis due to the dysregulation of its target genes. ${ }^{10}$ The current study was conducted to investigate the miR-1962a SNP rs11614913 contribution to the risk of CLL development in the Egyptian patients newly diagnosed with CLL.

Our results revealed a significant prevalence of the CC genotype and $\mathrm{C}$ allele in CLL patients. The TT and TC genotypes were significantly predominant in the control group compared to CLL patients.Subjects with $\mathrm{CC}$ genotype were found to be 11 times more prone to have CLL and subjects with the $\mathrm{C}$ allele were found to have a 3.38-fold increased risk for developing CLL compared to those with the protective $\mathrm{T}$ allele. These findings suggest the implication of the $\mathrm{C}$ allele in the risk of developing CLL in Egyptian patients. The molecular mechanism by which the risk allele predisposes to CLL remains unclear. However, a previous study revealed that miR$196 \mathrm{a}-2$ is involved in a number of biological pathways related to carcinogenesis including tumor suppressors and oncogenes. They also found that the introduction of pmiR-196a-C result in down-regulation of tumor suppressors and up regulation of oncogenes which confirmed the overall oncogenic activity, and the diminished regulatory activity of miR-196a-T. These findings are consistent with our regarding the protective role for the T allele of the SNP. ${ }^{9}$

The association between miR-196a2 SNP rs11614913 and CLL was studied in other populations and revealed different results. On the contrary to our results, Martin-Guerrero et al., reported thatmiR196a2 rs11614913 homozygote TT showed an increased risk of CLL in the Caucasian population of Spain..$^{10}$ Meanwhile, in the European
Caucasians, Jamroziak et al., ${ }^{11}$ studied the rs 11614913 SNP in a total of 561 including 195 patients with CLL, 166 patients with monoclonal B lymphocytosis (MBL) and 200 healthy control individuals and the Logistic regression analysis did not detect any significant associations of CLL or MBL with studied genotypes or alleles. ${ }^{11}$

However, the $\mathrm{C}$ allele was found to be associated with the risk of several cancers. In the Chinese population, a study was carried out by Tong et al, suggested that miR-196a 2 rs $11614913 \mathrm{C}$ allele may increase the risk of childhood ALL in the Chinese population. ${ }^{12}$ Also, Tian T, et al. and Chu et al., have found that CC genotype of miR196a2 SNP rs11614913 to be associated with increased risk for lung cancer and breast cancer respectively. ${ }^{13,14}$

Among Turkish population, Akkız $\mathrm{H}$ et al, reported that the $\mathrm{CC}$ genotype was associated with increased risk of hepatocellular carcinoma (HCC) development. ${ }^{15}$ Also, in the Polish population, Marcin Skalski, et al., reported a significant association between the CC genotype and laryngeal squamous cell carcinoma. ${ }^{16}$ Moreover, YuHui Wang performed a meta-analysis of 21 studies involving 26,018 subjects for miR-196a 2 rs 11614913 polymorphism. They reported that the $\mathrm{T}$ allele or its carriers were associated with decreased risk for breast cancer, lung cancer, colorectal cancer in overall analysis in Asian and Caucasian population. ${ }^{17}$ Similarly, Wang J, et al., carried out a meta-analysis included 32 studies and foundthat the TT genotype was associated with decreased cancer risk compared to the CC genotype in the Chinese population. ${ }^{18}$ On the other hand, opposite results were reported by Toraih EA et al., in the Egyptian population. They found an association between the $\mathrm{T}$ allele and the risk of colorectal, esophageal, skin, lung, thyroid, and renal cancer development in Egyptian cancer patients. The controversies between our and their results may be attributed to the organ-specific effects. ${ }^{19}$ Another meta-analysis conducted by Xu et al, showed that rs 11614913 in miR-196a2 TT genotype and T allele contributes to susceptibility to lung cancer cancer. ${ }^{20}$ However, Catucci and Jedlinski et al., ${ }^{21,22}$ could not identify any statistical association between miR-196a2 rs 11614913 genotypes and risk of breast cancer in German Italian and European Caucasian populations respectively.

To our knowledge, this the first study to investigatemiR-1962a rs3217927 SNP in Egyptian patients with CLL. The difference in genotypes and alleles frequencies of miR-196a2 rs11614913 between Egyptian, Chinese, German Italian, European Caucasian and other populations could be attributed to the genetic heterogeneity among 
these different populations and different methods used in genotyping. Interestingly, even when the same allele was associated with risk, this was the predominant allele in the same population, while it was the minor allele in others. Despite contradictions regarding the association of thers11614913 genotype and allele variants with cancer risk, it seems that rs11614913 SNP may play an important role in cancer development in all populations.

\section{Conclusion}

From our results, we could conclude that miR-1962a rs3217927 polymorphism have a phenotypic impact as the $\mathrm{C}$ allele may be implicated in the risk of CLL development in Egyptian patients. It is involved in one way or another in the pathogenesis of CLL and could be a novel candidate for translational research by further larger mechanistic studies.

\section{Acknowledgments}

None.

\section{Conflicts of interest}

Authors declare no conflict of interest.

\section{References}

1. Bartel DP. MicroRNAs: genomics, biogenesis, mechanism, and function. Cell. 2004;116(2):281-297.

2. Alshatwi AA, Shafi G, Hasan TN, et al. Differential expression profile and genetic variants of microRNAs sequences in breast cancer patients. PLoS One. 2012;7(2):e30049.

3. Ryan BM, Robles AI, Harris CC. Genetic variation in microRNA networks: the implications for cancer research. Nat Rev Cancer. 2010;10(6):389-402.

4. Landi D, Gemignani F, Landi S. Role of variations within microRNAbinding sites in cancer. Mutagenesis. 2012;27(2):205-210.

5. Chen C, Zhang Y, Zhang L, et al. MicroRNA-196: critical roles and clinical applications in development and cancer. $J$ Cell Mol Med. 2011;15(1):14-23.

6. Calin GA, Ferracin M, Cimmino A, et al. A MicroRNA signature associated with prognosis and progression in chronic lymphocytic leukemia. The New England journal of medicine. 2005;353(17):17931801 .

7. Wojcik SE, Rossi S, Shimizu M, et al. Non-codingRNA sequence variations in human chronic lymphocytic leukemia and colorectal cancer. Carcinogenesis. 2010;31(2):208-215.

8. Goldin LR, Slager SL, Caporaso NE. Familial chronic lymphocytic leukemia. Current opinion in hematology. 2010;17(4):350-355.

9. Hoffman AE, Zheng T, Yi C, et al. microRNA miR-196a-2 and breast cancer: a genetic and epigenetic association study and functional analysis. Cancer research. 2009;69(14):5970-5977.
10. Martin-Guerrero I, Gutierrez-Camino A, Lopez-Lopez E, et al. Genetic variants in miRNA processing genes and pre-miRNAs are associated with the risk of chronic lymphocytic leukemia. PLoS One. 2015;10(3):e0118905.

11. Krzysztof Jamroziak JS, Andy Rawstron, Zofia Szemraj-Rogucka, Polymorphisms of Mir-34b/c, Mir-146a and Mir-196a-2 and Predisposition to Chronic Lymphocytic Leukemia and Monoclonal BCell Lymphocytosis. Blood. 2011: 118:4585.

12. Tong N, Xu B, Shi D, et al. Hsa-miR-196a2 polymorphism increases the risk of acute lymphoblastic leukemia in Chinese children. Mutat Res. 2014;759:16-21.

13. Tian $\mathrm{T}$, Shu $\mathrm{Y}$, Chen $\mathrm{J}$, et al. A functional genetic variant in microRNA-196a2 is associated with increased susceptibility of lung cancer in Chinese. Cancer epidemiology, biomarkers \& prevention. 2009;18(4):1183-1187.

14. Chu H, Wang M, Shi D, et al. Hsa-miR-196a2 Rs11614913 polymorphism contributes to cancer susceptibility: evidence from 15 case-control studies. PLoS One. 2011;6(3):e18108.

15. Akkiz H, Bayram S, Bekar A, et al. A functional polymorphism in premicroRNA-196a-2 contributes to the susceptibility of hepatocellular carcinoma in a Turkish population: a case-control study. Journal of viral hepatitis. 201;18(7):e399-e407.

16. Skalski M, Ustaszewski A, Jaskiewicz K, et al. Single nucleotide polymorphism rs11614913 associated with CC genotype in miR$196 \mathrm{a} 2$ is overrepresented in laryngeal squamous cell carcinoma, but not salivary gland tumors in Polish population. Journal of applied genetics. 2018;59(3):301-304.

17. Wang $\mathrm{YH}, \mathrm{Hu} \mathrm{HN}$, Weng H, et al. Association between Polymorphisms in MicroRNAs and Risk of Urological Cancer: A Meta-Analysis Based on 17,019 Subjects. Frontiers in physiology. 2017;8:325.

18. Wang J, Wang Q, Liu H, et al. The association of miR-146a rs2910164 and miR-196a2 rs11614913 polymorphisms with cancer risk: a metaanalysis of 32 studies. Mutagenesis. 2012;27(6):779-788.

19. Toraih EA, Fawzy MS, Mohammed EA, et al. MicroRNA-196a2 Biomarker and Targetome Network Analysis in Solid Tumors. Molecular diagnosis \& therapy. 2016;20(6):559-577.

20. $\mathrm{Xu} \mathrm{W,} \mathrm{Xu} \mathrm{J,} \mathrm{Liu} \mathrm{S,} \mathrm{et} \mathrm{al.} \mathrm{Effects} \mathrm{of} \mathrm{common} \mathrm{polymorphisms} \mathrm{rs11614913}$ in miR-196a2 and rs2910164 in miR-146a on cancer susceptibility: a meta-analysis. PLoS One. 2011;6(5):e20471.

21. Catucci I, Yang R, Verderio P, et al. Evaluation of SNPs in miR146a, miR196a2 and miR-499 as low-penetrance alleles in German and Italian familial breast cancer cases. Human mutation. 2010;31(1):E1052-E1057.

22. Jedlinski DJ, Gabrovska PN, Weinstein SR, et al. Single nucleotide polymorphism in hsa-mir-196a-2 and breast cancer risk: a case control study. Twin research and human genet. 2011;14(5):417-421. 